\title{
INSTRUMENTO TEMÁTICO PARA AVALIAÇÕES SISTÊMICAS DOS FATORES QUE INFLUENCIAM A RETENÇÃ̃O DE VOLUNTÁRIOS SOCIAIS NA ÁREA DA SAÚDE
}

\author{
Raquel Pavan Braz ${ }^{1}$ \\ Universidade Federal Fluminense \\ raquelpbraz@gmail.com \\ Gilson Brito Alves Lima ${ }^{2}$ \\ Universidade Federal Fluminense \\ glima@id.uff.br
}

\begin{abstract}
Resumo
Os voluntários são relevantes para as organizações de voluntariado, pois sem eles a continuidade dos serviços é afetada. Logo, este artigo propõe um instrumento gerencial temático que especifique as informações a serem abordadas nas avaliações sistêmicas sobre as dimensões e os fatores de influência do voluntariado na área da saúde, buscando entender as questões influenciadoras da sua retenção. A metodologia foi composta pela revisão sistemática da literatura e por um grupo focal. Concluíu-se pela existência de 36 fatores temáticos, organizados em quatro dimensões de influência: individual (sociodemográficos, saúde do voluntário, experiência anterior com a doença, experiência com o voluntariado, aprimoramento/autoestima, valores, compreensão, proteção, carreira, expectativa, conflito); familiar (responsabilidades, apoio/suporte, influência); social (socializar, compromisso social, reconhecimento, apoio/suporte, pertencimento, agente de mudança social) e organizacional (infraestrutura, serviços e atividades, carga-horária, seleção, recrutamento, capacitação, recursos humanos, incentivos, autonomia, comunicação interna, relações interpessoais, apoio organizacional, estressores, acompanhamento, relação com a comunidade, relações externas).
\end{abstract}

Palavras-chave: Voluntariado Social. Saúde. Fatores de retenção. Dimensões de influência. Sistematização.

\section{THEMATIC INSTRUMENT FOR SYSTEMIC EVALUATIONS OF FACTORS INFLUENCING THE RETENTION OF SOCIAL VOLUNTEERS IN THE HEALTH AREA}

\begin{abstract}
Volunteers are relevant to volunteer organizations because without them the continuity of services is affected. Therefore, this article proposes a thematic management instrument that specifies the information to be addressed in systemic assessments of the dimensions and factors of influence of volunteering in the health area, seeking to understand the issues that influence their retention. The methodology applied was composed by a systematic review of the literature and a focus group. The results reveal the existence of 36 thematic factors organized in four dimensions of voluntary influence: individual (sociodemographic, volunteer health, previous experience with the disease, experience with volunteering, improvement/self esteem, values, understanding, protection, career, expectation, conflict); family (responsibilities, support, influence); social (socializing, social commitment, acknowledgement, support, belonging, social change agent) and organizational (infrastructure, services and activities, scheduling, recruitment, training, human resources, incentives, autonomy, internal communication, interpersonal relations, organizational support, stressors, follow-up, community relations, external relations).
\end{abstract}

Keywords: Social Volunteering. Health. Retention factors. Dimensions of influence. Systematization.

\footnotetext{
${ }^{1}$ Escola de Engenharia - Mestrado Profissional em Sistemas de Gestão.
}

${ }^{2}$ Escola de Engenharia - Mestrado Profissional em Sistemas de Gestão.

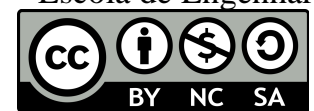




\section{INTRODUÇÃO}

O presente artigo faz parte de um estudo que teve como objetivo propor um instrumento gerencial temático para auxiliar na realização de pesquisas de avaliação sistêmica dos fatores influenciadores da retenção de voluntários sociais, na área da saúde. Este recorte representa a consolidação dos resultados das duas fases da investigação: revisão sistemática da literatura e grupo focal (BRAZ, 2019).

A manutenção da força de trabalho voluntária é essencial para a eficiência dos programas de voluntariado, que vem se destacando por agregar valor ao processo de humanização nos serviços de saúde (SILVA; CONCEIÇÃO; CHAGAS, 2017; RODRIGUES; MEYER JUNIOR; CRUZ, 2014). Além de criar condições favoráveis para a recuperação dos pacientes (SILVA; CONCEIÇÃO; CHAGAS, 2017). Ademais, há o pressuposto de que a utilização da força de trabalho voluntária favorece a sustentabilidade dos programas, tendo em vista serem menos onerosos em relação aos trabalhadores remunerados (TOPP et al., 2015).

O papel desenvolvido pelos voluntários é um fator importante para o desempenho dos hospitais, pois se torna estratégico no tocante à economia gerada, através da redução dos custos operacionais, ajudam na relação com a comunidade, melhorando a imagem da instituição, e agem como facilitadores na obtenção de novos recursos (MEYER JUNIOR; PASCUCCI; MURPHY, 2012). Tendo em vista sua importância e o espaço relevante no qual atuam, dentro das instituições de saúde, se faz necessário o conhecimento das questões motivacionais que compõem o processo de voluntariado, com o intuito de promover uma gestão mais eficiente, buscando gerar um impacto positivo no tocante à retenção dessa força de trabalho. (BECKHAUSER; DOMINGUES, 2017).

A resposta dessa problemática perpassa por inúmeras variáveis que influenciam a rotatividade, conforme destaca Ferreira (2012, p. 18) "Nos interstícios da análise a estas variáveis surge, então, a continuidade do serviço voluntário, como uma combinação das motivações com os fatores de gestão da organização que podem influenciar os indivíduos e com a satisfação desses mesmos indivíduos”. Nesse contexto, a fusão dos resultados deste estudo buscou responder à questão da pesquisa: Quais os fatores temáticos devem ser abordados, em um instrumento de pesquisa, de forma a permitir a avaliação sistêmica das questões que interferem na retenção dos voluntários sociais, na área da saúde?

O instrumento proposto servirá como um manual para a elaboração do formulário a ser aplicado diretamente nos voluntários da organização, especificando as temáticas que devem ser abordadas. Podendo ser utilizado para a elaboração de pesquisa para mapear os fatores de 
influência da permanência dos voluntários em um determinado programa de voluntariado ou, individualmente, no momento do desligamento do voluntário, como o intuito de entender quais foram os fatores que influenciaram na sua desistência.

$\mathrm{O}$ artigo se encontra estruturado em cinco sessões. A primeira é composta pela introdução, apresentando a importância do trabalho voluntário na área da saúde. A segunda aborda as teorias e modelos motivacionais do processo de voluntariado. A terceira apresenta a metodologia utilizada na pesquisa. A quarta traz a discussão sobre os dados encontrados na revisão sistemática e no grupo focal, apresentando o instrumento proposto e as limitações do estudo. Por fim, a quinta sessão inclui a conclusão e a proposta para estudos futuros.

\section{REVISÃO DA LITERATURA}

\subsection{TEORIAS MOTIVACIONAIS}

Inúmeras pesquisas sobre o voluntariado buscam entender as razões da motivação dos voluntários. Esta pesquisa reuniu algumas teorias abordadas, mas não buscou esgotar o assunto, tendo em vista as inúmeras teorias existentes. Dentre as diversas formas de abordagem, chama atenção a que divide as teorias de motivação em teorias de conteúdo e teorias de processo (CAMPBELL et al., 1970). As primeiras buscam identificar constructos individuais ou situacionais que influenciam a motivação humana, considerando a satisfação das necessidades como ponto central (TAMAYO; PASCHOAL, 2003). As teorias de processo analisam o mecanismo pelo qual ocorre a motivação, presumindo que o ambiente externo possa interferir no comportamento dos indivíduos, pois ele passa a decidir, de forma consciente, sobre a realização de suas atividades (LOBOS, 1975; PÉREZ-RAMOS, 1990).

Uma das teorias de conteúdo mais famosa é a "teoria das motivações humanas" de Maslow (1943), a qual relaciona a motivação à satisfação das necessidades humanas organizadas, hierarquicamente, em cinco níveis: fisiológicas, segurança, sociais, estima e autorealização (MASLOW, 1943). Outra teoria relevante é a "teoria dos dois fatores", desenvolvida por Herzberg (1987), na qual os aspectos que afetam a atitude no trabalho são divididos em fatores de motivação intrínseca e higiênicos, tendo como base a crença de que os motivos que tornam os indivíduos mais satisfeitos e motivados, no trabalho, são diferentes dos que provocam a insatisfação, mas não opostos (HERZBERG, 1987).

A "teoria das trocas sociais" representa um exemplo de teoria de processo. Construída com base em conceitos econômicos, considera que as relações se formam, se mantêm ou se 
quebram de acordo com o custo-benefício envolvido (HOMANS, 1961). A Teoria Funcionalista também vem sendo constantemente empregada no campo do voluntariado e costuma adotar a diversidade de motivações e defender que as pessoas podem exercer as mesmas atividades ou tomar as mesmas atitudes por razões diferentes e objetivando servir a diversas funções psicológicas (CLARY et al., 1998; OMOTO; SNYDER, 1995).

Como alerta Ferreira (2012), as teorias motivacionais sobre trabalhadores remunerados não podem ser generalizadas e aplicadas aos trabalhadores voluntários de forma irrestrita, devido às diferenças das suas atividades. Ainda assim, representam um suporte importante na compreensão das expectativas e motivações na área do voluntariado.

\subsection{MODELOS DE ANÁLISE MOTIVACIONAL DO VOLUNTARIADO}

No campo do voluntariado, inúmeros modelos são apresentados com o objetivo de categorizar e explicar o que motiva o indivíduo a doar o seu tempo, sem qualquer remuneração, em prol de outros indivíduos (WIDJAJA, 2010). Algumas teorias tentam explicar as causas do voluntariado pelos atributos individuais dos voluntários, enquanto outras abordam, também, os recursos sociais e as atividades organizacionais (WILSON, 2000).

Estudos apontam diferentes sentimentos que podem encorajar atos de voluntariado. $\mathrm{O}$ altruísmo, por exemplo, é considerado um dos principais estímulos para o trabalho voluntário (SARTI et al., 2017). Cnaan e Goldberg-Glen (1991), argumentam que a relação entre motivos altruístas e egoístas resulta em motivadores para ações voluntárias, desenvolvendo o modelo de escala unidimensional. O modelo bidimensional, de Frisch e Gerrard (1981), procura separar esses dois conceitos. Já o modelo tridimensional considera motivos altruístas, sociais e materiais como fatores motivacionais do voluntariado (WIDJAJA, 2010; AFARI-ASIEDU et al., 2018).

Com base na teoria funcionalista, surgiram análises mais complexas como o modelo multidimensional, de Clary et al. (1998), o qual identificou seis funções motivacionais: valores, compreensão, social, carreira, protetora e aprimoramento/autoestima. A mesma teoria serviu como sustentação para os estudos de Omoto e Snyder (1995), que consideraram o trabalho voluntário como um processo subdividido em três estágios temporais (antecedentes, experiências e consequências), aduzindo que os fatores motivacionais podem mudar, com o tempo, devido ao amadurecimento do programa de voluntariado (ALAM; OLIVEIRA, 2014). 


\section{METODOLOGIA}

Inicialmente, foi realizada a revisão sistemática da literatura que buscou mapear as dimensões e os fatores abordados nas pesquisas sobre a retenção de voluntários sociais na área da saúde, realizadas nos últimos 10 anos, (BRAZ; LIMA, 2019, [no prelo]). Para essa fase foi utilizada a adaptação da recomendação PRISMA - Principais Itens para Relatar Revisões sistemáticas e Meta-análises (MOHER et al., 2015), que resultou na seleção de 13 artigos.

Os dados obtidos na análise desses artigos foram categorizados, segundo critérios semânticos, buscando agrupar os fatores que estivessem no mesmo campo temático (BARDIN, 1977), o que permitiu a elaboração de dois formulários. O primeiro consistiu na reunião das dimensões de influência do voluntariado e o segundo na elaboração do rol dos fatores temáticos abordados nas pesquisas de retenção. Ambos foram estruturados segundo a escala do tipo Likert com o objetivo de medir o grau de importância desses dados. A escalafoi estruturada com cinco pontos de avaliação (1 - Sem importância, 2 - Pouca importância, 3 - Razoavelmente importante, 4 - Importante, 5 - Extremamente importante). Sua aplicação ocorreu através da elaboração de um grupo focal.

A estruturação do grupo focal seguiu a sistemática desenvolvida por Vergara (2015) e Vergara (2016). O grupo foi composto por seis participantes, considerados especialistas no assunto. Todos estavam na faixa entre 44 e 78 anos de idade. A maioria era do sexo feminino (cinco), enquanto apenas um participante do sexo masculino. Em termos de ocupação, um participante era fisioterapeuta, um professor, um autônomo e três estavam aposentados, tendo exercido atividades de famacêutico-bioquímico, autônomo e advogado. No tocante à experiência, dois participantes apresentaram mais de 30 anos em atividades de voluntariado, três estavam entre 20 a 30 anos e o último, há menos de 10 anos. Todos alegaram desenvolve, semanalmente, atividades voluntárias. Com a autorização dos participantes, o áudio do debate foi registrado com gravador de voz, possibilitando sua transcrição na íntegra.

\subsection{DADOS CONSIDERADOS PARA A CONSTRUÇÃO DO INSTRUMENTO TEMÁTICO}

As dimensões e os fatores temáticos, inseridos no instrumento proposto, foram selecionados segundo as regras de cortes estipuladas para cada fase. Sendo necessário que, no mínimo, uma das exigências fosse cumprida. Essa sistemática está disposta na Figura 1. 
Figura 1 - Sistemática da inclusão dos fatores no instrumento proposto

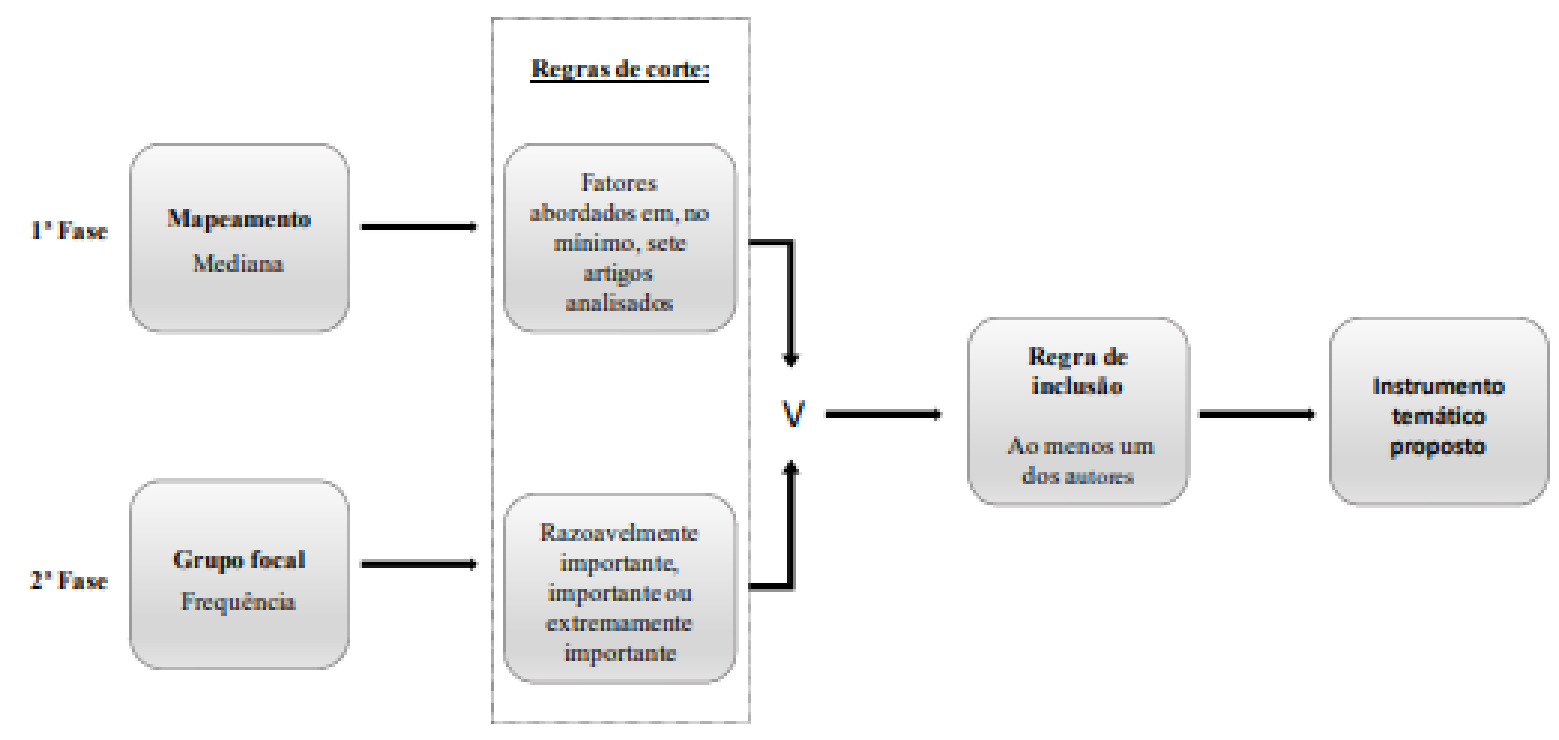

Fonte: Elaboração propria

Conforme a Figura 1, para os dados a serem considerados na fase do mapeamento, aplicou-se a mediana do número de artigos da revisão sistemática. Neste estudo foram considerados 13 artigos, o que gerou uma mediana de número 7. Os fatores temáticos que apresentaram abordagem a partir desse valor foram considerados para a inserção no instrumento proposto. Para os dados do grupo focal, dividiu-se a escala likert em dois grupos. O Grupo 1 reuniu os níveis "sem importância" ou "pouca importância", o Grupo 2, "razoavelmente importante", "importante" e "extremamente importante". Foram considerados os fatores e as dimensões que apresentaram maior frequência localizada nesse último grupo. Nos casos de empate, optou-se por considerá-los como válidos para o instrumento.

\section{ANÁLISE E DISCUSSÃo DE RESULTADOS}

\subsection{RESULTADOS DA REVISÃO SISTEMÁTICA}

O mapeamento dos artigos analisados detectou a existência de cinco dimensões de influência: individual, familiar, social, organizacional e política. Cada uma delas é composta por um rol de fatores temáticos, conforme estruturado na Figura 2. 
Figura 2 - Resultado obtido na fase da revisão sistemática

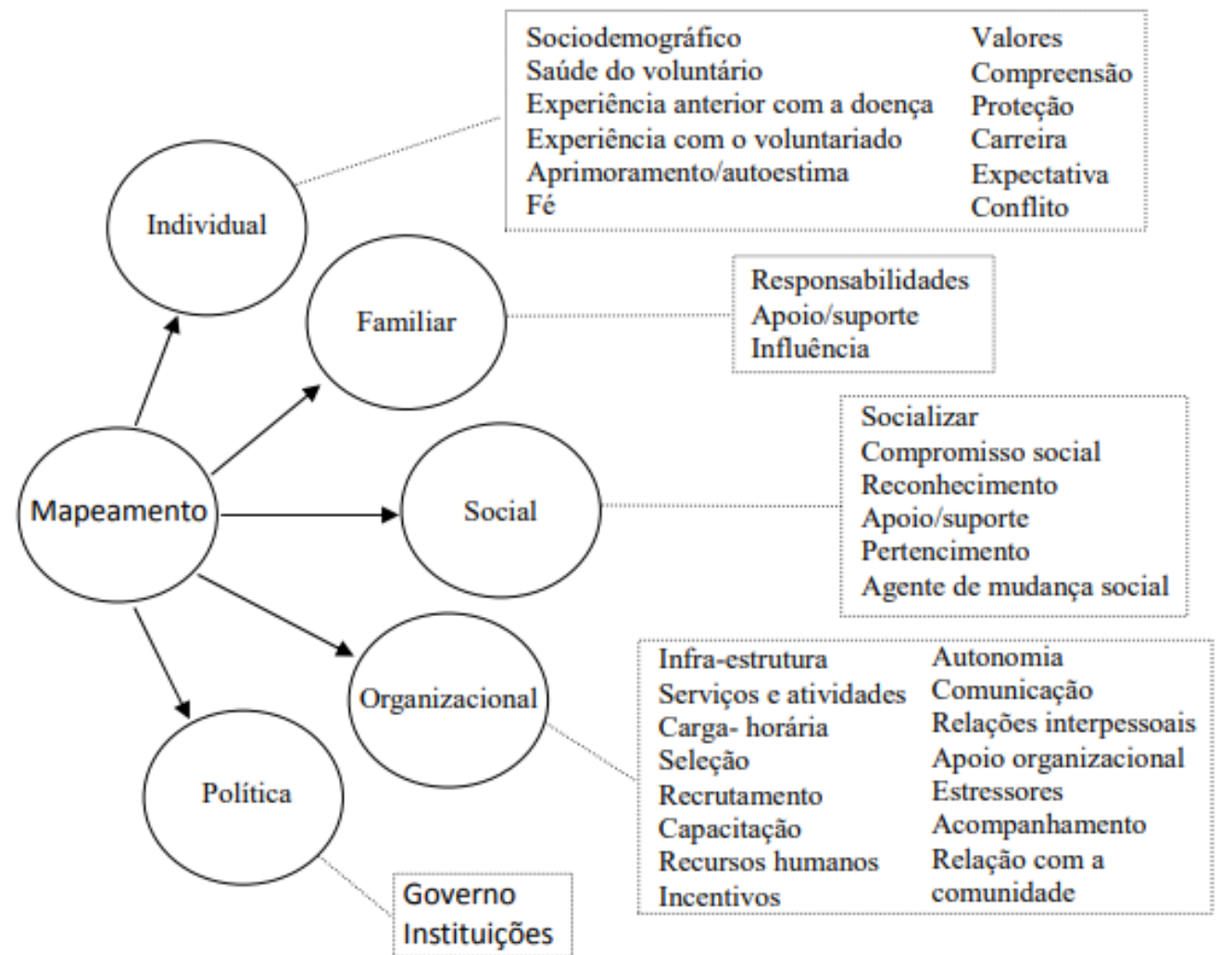

Fonte: Elaboração própria

Conforme Figura 2, a dimensão individual é composta por fatores temáticos divididos em sociodemográficos, saúde do voluntário, experiência anterior com a doença, experiência com o voluntariado, aprimoramento/autoestima, fé, valores, compreensão, proteção, carreira, expectativa e conflito. A dimensão familiar apresentou temas relacionados às responsabilidades familiares, ao apoio/suporte e a influência na decisão do voluntário. Em relação à dimensão social, foram mapeados fatores ligados à necessidade de socializar, ao comprometimento social, ao reconhecimento, ao apoio/suporte social, ao pertencimento à comunidade e ao desejo de ser um agente de mudança social.

A dimensão organizacional foi a que apresentou o maior número de temas abordados, totalizando 15 fatores temáticos, sendo eles: infraestrutura, serviços e atividades, carga- horária, seleção, recrutamento, capacitação, recursos humanos, incentivos, autonomia, comunicação, relações interpessoais, apoio organizacional, estressores, acompanhamento e relação com a comunidade. A dimensão política foi dividida em governo einstituições. 


\subsection{RESULTADOS DO GRUPO FOCAL}

Passando-se à análise dos dados quantitativos, através da aplicação dos dois questionários de grau de importância e utilizando-se a regra de corte para os dados do grupo focal, constatou-se a importância das dimensões: individual, familiar, social/comunitário e organizacional. A dimensão política foi a única que não teve a sua relevância considerada.

Em relação aos fatores temáticos, na dimensão individual foram selecionados os fatores: sociodemográfico (familiar, acadêmica, emprego, localidade), saúde do voluntário, experiência anterior com a doença, experiência com o voluntariado, aprimoramento/autoestima, valores, compreensão, proteção, expectativa e conflitos. A dimensão familiar abordou as responsabilidades, o apoio/suporte e a influência dos familiares. Já na dimensão social estiveram presentes: socializar, compromisso social, apoio/suporte, pertencimento e agente de mudança; A dimensão organizacional: infraestrutura, serviços e atividades, carga-horária, seleção, recrutamento, capacitação, recursos humanos, autonomia, comunicação, relações interpessoais (contato, apoio, retorno), apoio organizacional, estressores, acompanhamento e relação com a comunidade. Por fim, os fatores governo e instituições do nível político.

\section{3 INSTRUMENTO PROPOSTO}

O instrumento gerencial proposto surge a partir da consolidação dos resultados da revisão sistemática e do grupo focal, após a aplicação de suas respectivas regras de corte. Para fins de inclusão, foram consideradas as dimensões: individual, familiar, social/comuniário e organizacional. A dimensão política não foi selecionada, pois não preencheu nenhuma regra de corte. Em relação aos fatores temáticos, a Figura 3 representa aqueles que foram recepcionados e a sua respectiva fase de inclusão. 
Figura 3 - Fatores considerados para inserção no instrumento proposto

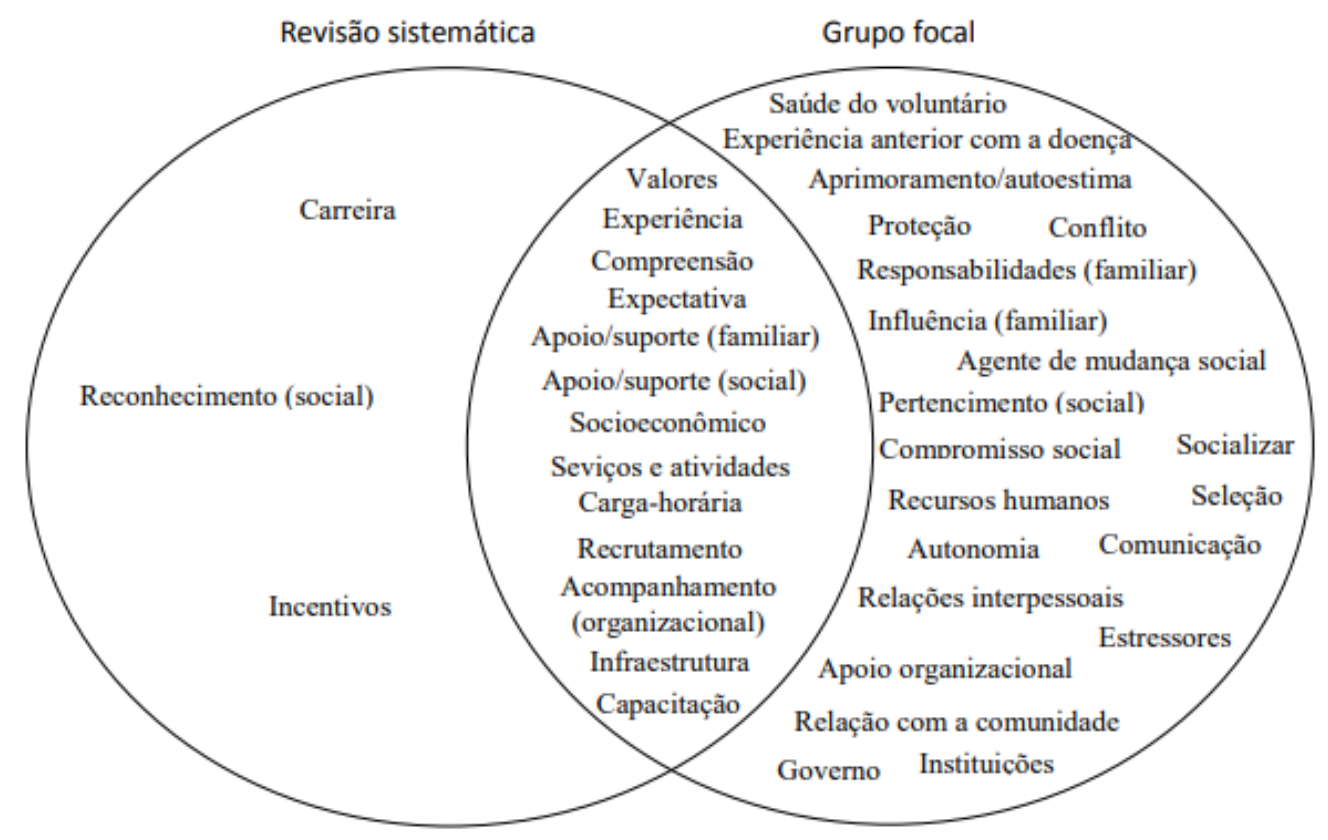

Fonte: Elaboração própria

Conforme observado na Figura 3, da esfera individual, os fatores sociodemográficos foram considerados importantes por consenso das duas fases. Dentre eles a idade foi um dos assuntos mais questionados. A literatura registra que a faixa etária pode representar um fator de interferência na permanência dos voluntários nos programas de voluntariado (ALAM; OLIVERAS, 2014). Chatio e Akweongo (2017), verificaram que alguns voluntários abandonaram as atividades voluntárias devido à idade avançada. De forma contrária à literatura, essa situação não foi considerada crítica pelos participantes do grupo focal.

A saúde e os casos de doenças do próprio voluntário foram considerados uma barreira (SANOU et al., 2016) e um dos motivos para interromper o voluntariado (CLAXTONOLDFIELD; CLAXTON-OLDFIELD, 2012). Valor parecido foi atribuído pelos participantes do grupo focal, que consideraram importante a questão da experiência anterior com a doença relacionada à instituição de saúde. Hyde et al. (2016), constataram que a maioria dos voluntários entrevistados conhecia pessoas que já haviam sido diagnosticadas com a doença anteriormente. "Eu tive um pouco dessa influência pelo sofrimento de duas pessoas da minha família, foi por isso (câncer) que a minha mãe morreu. Mas isso influenciou no sentido de que eu vi que eu posso evitar o sofrimento de outros, é uma influência.”, disse um dos especialistas. Outro membro do grupo é ex-paciente e expressou seu sentimento:

Eu, como já fui paciente, já tive câncer, pra mim, eu custei muito a retornar aquele ambiente. Eu acho que para eu ser voluntária numa enfermaria de câncer, eu, hoje, ainda não 
tenho condições. Entendeu? Eu ainda custei bastante. Sou voluntária, participo do outubro rosa, participo em várias situações assim, mas para estar lá de frente, eu acho que ainda é pesado. Eu como paciente.

No que tange a experiência com o voluntariado, o grupo focal atribuiu importância a essa questão. A literatura não recepcionou o tema de forma significativa, mas um fato que chamou a atenção foi a constatação de uma maior propensão da permanência dos voluntários que participam, concomitantemente, de vários programas de voluntariado. Em outras palavras, a experiência vivenciada em organizações diversas, durante o mesmo período, foi reconhecida como um fator que favorece a retenção do voluntário (ALAM; OLIVERAS, 2014). As questões sobre o fator aprimoramento/autoestima, também, não apareceram muito na literatura estudada, mas o grupo focal entendeu que é pertinente sua presença na retenção dos voluntários.

O fator temático "valores" se apresentou relevante para as duas fases da pesquisa, o que demonstra que o atendimento das expectativas em relação aos valores individuais é importante para a satisfação dos voluntários (TOPP et al., 2015). Assim como o fator “compreensão", sobre o qual Clary et al. (1998), atribuiram o desejo de adquirir e exercitar novos conhecimentos e habilidades como motivadores para o desenvolvimento de atividades voluntárias. Singh et al. (2016), constataram que a maioria dos voluntários possuíam o desejo de adquirir mais conhecimentos específicos na área da saúde.

Questões relacionadas à temática proteção, segundo o conceito trazido por Clary et al. (1998), foram abordadas em um único estudo analisado. Porém, na avaliação do grupo focal, este fator ficou situado no grupo 2, o que o tornou elegível para a inserção no instrumento. Já em relação ao fator "carreira", a esperança de renda foi bem representada nos estudos analisados, baseada na possibilidade de remuneração, que se demonstrou um fator motivador para os voluntários (SANOU et al., 2016; ALAM; OLIVEIRA, 2014; ALAM; TASNEEM; OLIVERA, 2012), chegando a apresentar um fator crítico de abandono devido à falta de pagamento de salários regulares nesse tipo de atividade (SANOU et al., 2016).

$\mathrm{O}$ fator expectativa surgiu da possibilidade de abordagem do tema de forma aberta, não sendo restrita a um assunto específico. Sua importância foi ratificada tanto pela frequência de abordagem na literatura como pelos especialistas do grupo focal. Nos estudos desenvolvidos por Topp et al. (2016), os participantes demonstraram descontentamento com vários fatores, tendo em vista que suas expectativas não estavam sendo atendidas pelo programa de voluntariado.

No fator "conflito", a competição com outros empregos ou programas se demonstrou significativa para a retenção (ALAM; OLIVERAS, 2014). Sanou et al. (2016), confirmaram 
que o trabalho voluntário pode representar um grande desafio, tendo em vista que prejudica a possibilidade do exercício concomitante com outro tipo de atividade remunerada, afetando a renda dos voluntários. Esse foi um dos motivos mais citados, nos estudos realizados por Ludwick et al. (2014), como sendo a causa de abandono dos programas de voluntariado. O conflito de tempo, também, representa um fator complicado na tentativa de conciliar as atividades voluntárias com as pessoais dos voluntários, resultando, muitas vezes, no abandono do trabalho voluntário (CHATIO; AKWEONGO, 2017; ALAM, OLIVERA, 2014; LUDWICK et al., 2014). Sua importância foi reconhecida pelo grupo focal.

Dos fatores mapeados, o econômico, a religião e a fé não foram selecionados para compor o instrumento proposto, pois não preencheram nenhuma regra de corte. Porém, alguns estudos registraram que as necessidades econômicas dos voluntários podem representar uma barreira para a realização desse tipo de atividade (TOPP et al., 2105). Em relação à religião, durante o grupo focal, um dos especialistas relatou que "Era uma forma de se chegar. [...] Mas no trabalho voluntário isso é um dificultante absurdo, eu acho. Nesse sentido do preconceito que ele gera.", o que pode sugerir que a religião, em alguns momentos, pode ser considerada como uma forma de estímulo para a adesão aos programas de voluntariado, mas não para a sua permanência, podendo se tornar, até mesmo, um impecílio.

Os fatores da dimensão familiar não obtiveram abordagem significativa na literatura. Apenas o fator apoio/suporte preencheu a regra de corte das duas fases. Estudos concluíram que a desaprovação da família é um fator de risco para a retenção (ALAM; OLIVERA, 2014; ALAM; TASNEEM; OLIVERA, 2012). O grupo focal reconheceu a importância das responsabilidades familiares, corroborando com estudos que concluíram que os indivíduos, que não apresentavam conflitos entre a prática do voluntariado e suas responsabilidades, possuíam duas vezes mais probabilidade de permanecer (ALAM; TASNEEM; OLIVERA, 2012).

Com relação à influência, há indícios de que muitos indivíduos passam a desenvolver atividades voluntárias, porque algum amigo ou familiar já desenvolve esse tipo de trabalho (HYDE et al., 2016). No entanto, a discussão do grupo focal seguiu por uma linha contrária, refletindo a divergência entre os especialistas. Fato que resultou em empate na frequência dos grupos de importância, mas foi suficiente para preencher a regra de corte.

Na dimensão social, o único fator temático que resultou do consenso entre o que versa a literatura e a prática do grupo focal foi o apoio/suporte social. O reconhecimento social preencheu a regra de corte estipulada para o tratamento dos dados advindos da literatura, já o restante dos fatores derivou da realização do grupo focal. 
A literatura demonstra que socializar é encarado como um fator motivacional relevante no processo de voluntariado (HYDE et al., 2016; SINGH et al., 2016), embora sua abordagem tenha sido baixa. O respeito (SINGH et al., 2016), e o reconhecimento da comunidade foram considerados fatores que podem influenciar os voluntários, assim como o status social (AFARIASIEDU et al., 2018; SANOU et al., 2016). Chatio e Akweongo (2017), constataram que a falta de respeito e de reconhecimento foram as razões pelas quais alguns indivíduos pararam de trabalhar como voluntários. Em contrapartida, Claxton-oldfield e Claxton-oldfield (2012), apuraram que o reconhecimento não representou um fator significativo para os voluntários que atuavam em atividades de cuidados paliativos. Resultado parecido ao constatado nos dados do grupo focal.

O envolvimento da comunidade é considerado um fator crítico para a retenção (STRACHAN et al., 2012). Paralelamente, o apoio da comunidade pode apresentar um fator desestimulante (SANOU et al., 2016), ou um motivador para a retenção (CHATIO; AKWEONGO, 2017). Segundo Alam, Tasneem e Olivera (2012), os voluntários que receberam o apoio e a aprovação da comunidade tinham quase três vezes mais chances de permanecer do que os que não experimentaram da mesma situação.

Há, ainda, o sentimento de se tornar um agente de mudança que, por vezes, gera a motivação necessária para participação em programas de voluntariado na área da saúde (HYDE et al., 2016; SINGH et al., 2016; TOPP et al., 2015). Esse fator foi constatado em, apenas, três dos artigos analisados, mas os dados quantitativos do grupo focal atribuiram a ele o grau de importância necessário para ser inserido na versão final do instrumento proposto.

Em relação aos fatores organizacionais, os que apresentaram consenso nos resultados foram: infraestrutura, serviços e atividades, carga-horária, recrutamento, capacitação e acompanhamento. $\mathrm{O}$ fator relativo aos incentivos preencheu a regra de corte para os dados da literatura. Os fatores referentes à seleção, recursos humanos, autonomia, comunicação, relações interpessoais, apoio organizacional, estressores e relação com a comunidade foram considerados com base na regra de corte para os dados provenientes do grupo focal.

No que se refere à infraestrutura, a falta de suprimentos para realizar o trabalho foi diagnosticada como um fator desestimulante (SANOU et al., 2016), podendo representar uma importante barreira para os voluntários (MAYS et al., 2017; TOPP et al., 2015), e resultar em

abandono das atividades voluntárias (CHATIO; AKWEONGO, 2017). Pois eles tendem a facilitar o desenvolvimento das atividades, favorecendo a permanência do voluntário (MAYS et al., 2017). Essa importância também foi verificada nos dados do grupo focal. 
Em relação aos serviços e atividades, o voluntariado na área da saúde, muitas vezes, ocorre devido a sua natureza, na intenção de ajudar na prestação dos serviços de saúde por ser um trabalho valioso para a comunidade (AFARI-ASIEDU et al., 2018, LUDWICK et al. (2014). Porém, dentre as temáticas que envolvem esse fator, algumas podem representar desafios, como o volume de trabalho (SANOU et al. (2016), tornando-o importante na discussão sobre a retenção dos voluntários. Entendimento compatível com as alegações trazidas pela literatura e os dados obtido através do grupo focal.

Outra questão importante é representada pela carga-horária. Os questionamentos a seu respeito, constantemente, sobrecaem no número de horas trabalhadas (AFARI-ASIEDU et al., 2018; MOGHADDAM; ALLAHVERDIPOUR; MATLABI, 2018) e a frequência (MAYS et al., 2017; TOPP et al., 2015), com a qual o voluntário desenvolve suas atividades. A tentativa de analisar se a quantidade de trabalho realizada está de acordo com a carga que o voluntário consegue absorver é proveniente da constatação de que o excesso de trabalho pode apresentar um grande desafio para a retenção dos voluntários (SANOU et al., 2016). Compreensão consoante com a percepção da literatura e do grupo focal.

O recrutamento é reconhecido, por alguns estudos, como um fator crítico para a motivação e retenção dos voluntários. Neste estudo, ele foi considerado tanto pela literatura quanto pelo grupo focal. Resultado diferente do qual obteve a questão da seleção de voluntários, só sendo recepcionada pelo grupo focal. Durante o debate foi levantada a necessidade de elaboração de uma seleção transparente, de forma a evitar frustração futura para os novos voluntários, podendo gerar desistências, como relata um dos especialistas:

A gente chega com muita ideia pré-concebida: 'Ah, eu vou para esse trabalho, eu quero fazer isso'. Chega lá, se você não for orientado, você vai sofrer uma frustração terrível, né. Você tem que saber, ou em uma entrevista ou em algum lugar, em algum momento, em uma seleção. 'Olha, você está aqui e o trabalho é assim. Você vai ser preparado para esse trabalho'. Porque as pessoas idealizam o tempo todo. A idealização do trabalho pode ser de uma frustração terrível e ela, com a maior força de vontade, vai querer fazer do jeito que ela pensou, imaginou e isso vai ser um problema.

A implantação de ações de treinamentos, seja para desenvolver habilidades básicas ou para atualização dos conhecimentos, também demonstrou ser um fator importante para a retenção e motivação dos voluntários (AFARI-ASIEDU et al., 2018; CHATIO; AKWEONGO, 2017; SANOU et al., 2016; LUDWICK et al., 2014; ALAM; TASNEEM;

OLIVERA, 2012; STRACHAN et al., 2012). Pois, aqueles que estão regularmente em contato com programas de treinamento costumam apresentar maior probabilidade de retenção 
(SANOU et al., 2016; ALAM; OLIVERA, 2014). O entendimento da literatura coincidiu com o do grupo focal, o qual ratificou a necessidade e a importância das ações de treinamento para os voluntários. "Eu acho que é importante. Você vai realizar uma atividade, não é chegar... você pode estar com todo o carinho, com toda a vontade, mas tem que saber como fazer e fazer da melhor forma possível. Eu acho que a Instituição tem que prover isso", disse um dos especialistas durante a discussão do grupo focal.

O fator temático recursos humanos, categorizado por este estudo, buscou reunir fatores que versão sobre a gestão direcionada aos indivíduos que fazem parte da instituição como voluntários. A forma como a gestão de recursos humanos é estruturada e outras questões pertencentes a esse campo semântico foram mapeadas. A abordagem sobre a temática na literatura não foi relevante, mas os dados quantitativos averiguados no grupo focal indicaram a pertinência da sua avaliação.

Os incentivos, tanto financeiros quanto os não financeiros, são fatores abordados com frequência nos estudos sobre retenção na área do voluntariado. Em pesquisa realizada por Strachan et al. (2012), essa temática foi a mais discutida entre os participantes, sendo considerada crítica para o processo de voluntariado. Já Afari-Asiedu et al. (2018), concluíram sobre a ação facilitadora desses fatores para a retenção dos voluntários.

Em alguns estudos os incentivos financeiros apresentaram maior ligação com a retenção de voluntários (ALAM; TASNEEM; OLIVERA, 2012; MAYS et al., 2017), como os subsídios para transporte, que foi um dos fatores mais abordados, dentro dessa temática, podendo interferir no processo de voluntariado, agindo de forma desestimulante para os voluntários (MAYS et al., 2017; SANOU et al., 2016; SINGH et al., 2016).

No entanto, Moghaddam, Allahverdipour e Matlabi (2018), alertaram para a possível perda da natureza voluntária caso os voluntários passem a receber salários regulares. Preocupação compartilhada com os participantes do grupo focal. Um dos especialistas se pronunciou dizendo "Eu sou contra incentivo monetário". Contudo, os próprios participantes atentaram para um olhar diferente, sinalizando que a organização deve dar o suporte necessário para que o voluntário possa desenvolver suas atividades. "Tem que ter esse olhar, porque já perdemos muitos voluntários por conta disso. Porque não tem dinheiro e vai embora, ou vai para outro bairro longe para caramba.", disse outro especialista.

Os incentivos não financeiros, ou os chamados benefícios materiais, também tiveram um papel de destaque e externaram a importância de pequenos gestos para aumentar o senso de orgulho e valorização pessoal como, por exemplo, a distribuição de camisetas, crachá de identificação, emissão de certificados de participação em treinamentos (SANOU et al., 2016), 
e prêmios (CHATIO; AKWEONGO, 2017). Um dos especialistas indagou que "Incentivo é bom. Você chega e tem um cafezinho para você tomar, talvez.", outro completou "Um abraço junto com um bolinho, um cafezinho".

Sobre o fator "autonomia", a literatura não foi muito expressiva, mas o grupo focal demonstrou sua importância para os voluntários. A mesma lógica seguiu o fator relacionado à comunicação interna. O grupo chegou ao consenso de que é um fator de destaque para o desenvolvimento das atividades voluntárias. Segundo um dos especialistas "É a coisa mais difícil em qualquer atividade humana, não só no voluntariado. As pessoas têm uma dificuldade enorme de expressar o que estão pensando e pensam e falam de uma forma que não gostariam de falar". A falha na comunicação, pode resultar na construção de falsas expectativas em relação à natureza voluntária das atividades e gerar frustração entre os voluntários, aumentando os níveis de evasão (ALAM; TASNEEM; OLIVERA, 2012).

Outra questão de tensão está relacionada às relações interpessoais. Chatio e Akweongo (2017), inferiram que, segundo a percepção dos profissionais de saúde, o reconhecimento deles em relação ao trabalho dos voluntários e a constante interação entre eles e a equipe de saúde configuram fatores motivacionais importantes para o processo de desenvolvimento das atividades voluntárias. Outros fatores motivacionais são representados pelo apoio da equipe de saúde no desenvolvimento das atividades e do trabalho em equipe (MOGHADDAM, ALLAHVERDIPOUR; MATLABI, 2018; LUDWICK et al., 2014). Essa importância foi expressa por um dos especialistas como sendo "Extremamente importante. [...] como diz uma amiga, não é um grupo, é um time, é uma equipe. Então tem que estar todo mundo junto”.

Outro tipo de apoio relevante para o processo de voluntariado é o praticado pela organização que, para fins deste estudo, abrange o apoio dado aos voluntários com cunho mais pessoal e subjetivo como o apoio emocional, o psicológico e o espiritual. Por vezes, esse suporte ocorre através de reuniões entre os voluntários e seus gestores, sendo considerada, pela literatura, como possíveis fatores motivacionais para a continuidade do trabalho voluntário (CHATIO; AKWEONGO, 2017). Embora sua abordagem tenha sido pouco verificada nos artigos analisados, resultado mais expressivo foi encontrado nos dados provenientes do grupo focal, tendo sua frequência de respostas localizada no grupo 2.

O cansaço e o esgotamento emocional foram citados como fatores influenciadores no abandono do voluntariado, nos estudos de Claxton-Oldfield e Claxton-Oldfield (2012), representam alguns dos estressores desse tipo de atividade. Embora o campo de estudo esteja centrado na área da saúde que, pela natureza das atividades, muitas vezes, apresenta situações delicadas, a literatura não tratou de forma siginificativa o assunto. A discussão do 
grupo focal ratificou sua existência. Um dos participantes alegou que "No hospital, sendo voluntário, muitos (estressores)", sendo completado por outro membro do grupo:

Você já tem que avaliar isso antes de entrar. Sabendo que, dependendo do momento que você está vivendo, você pode sentir mais ou menos impacto. Tem gente que: 'não, esse trabalho eu não posso fazer.'. Você achou que podia, mas no momento você está mais fragilizado e aí você sente, balança ali, né. Balança e pode até desistir em algum momento.

Dentre os fatores de acompanhamento, a questão da supervisão foi constatada como sendo um fator significativo para a retenção dos voluntários (CHATIO; AKWEONGO, 2017; LUDWICK et al., 2014). Nos estudos realizados por Strachan et al. (2012), observou-se que muitos participantes consideraram a supervisão como sendo o fator mais importante no processo de motivação dos voluntários, por proporcionar um sentimento de pertencimento e aumentar a conexão com o programa.

Para o fator temático "relação com a comunidade", foram considerados os meios de comunicação pelos quais se estrutura a relação entre a organização e a sociedade e o processo de informação sobre o papel do voluntário, que se apresentam como importantes, pois ajudam na mobilização da comunidade (MOGHADDAM; ALLAHVERDIPOUR; MATLABI, 2018). Neste estudo, foi constatada baixa abordagem sobre a temática na literatura, estando presente em três estudos analisados, porém o grupo focal concluiu pela importância dessa relação para a manutenção do voluntário nos programas de voluntariado.

Os fatores classificados na dimensão política são aqueles referentes à relação entre os programas de voluntariado e os governos ou outras instituições. Essa temática foi mapeada em apenas três artigos, porém sua importância foi constatada pelo grupo focal, o que possibilitou sua inclusão no instrumento proposto. Contudo, considerando que a dimensão política não atingiu os requisitos para as regras de corte, optou-se por alocar esses fatores na dimensão organizacional, tendo em vista eles possuirem relação direta com a instituição.

Quando indagados sobre a existência de outros fatores ou dimensões não mencionadas, o grupo ponderou que muito havia sido discutido durante o debate. Um dos participantes indagou que "não existe certo e errado e é tudo tão subjetivo". Logo, não foram identificados novos fatores ou novas dimensões durante a discussão. 
Quadro 1 - Instrumento gerencial temático para pesquisas sobre retenção de voluntários na área da saúde

\begin{tabular}{|c|c|c|c|}
\hline $\begin{array}{c}\text { Dimensões } \\
\text { de } \\
\text { Influência }\end{array}$ & \multicolumn{2}{|c|}{ Fatores temáticos de Influência } & $\begin{array}{c}\text { Espaço livre para a formulação de questões } \\
\text { dentro de cada tema (para uso das Instituiçõos) }\end{array}$ \\
\hline \multirow{15}{*}{ 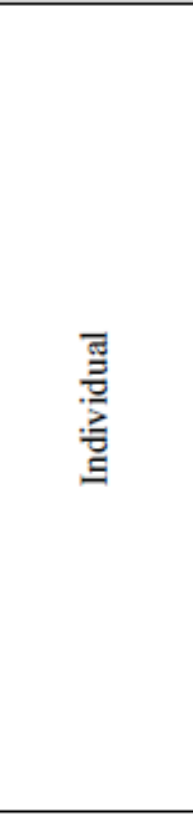 } & \multirow{5}{*}{ 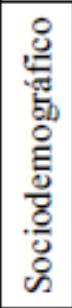 } & Individual & \\
\hline & & Familiar & \\
\hline & & Acadêmico & \\
\hline & & Emprego & \\
\hline & & Localidade & \\
\hline & \multicolumn{2}{|c|}{ Saúde do voluntário } & \\
\hline & \multicolumn{2}{|c|}{$\begin{array}{l}\text { Experiência anterior com a } \\
\text { doença }\end{array}$} & \\
\hline & \multicolumn{2}{|c|}{ Experiência } & \\
\hline & \multicolumn{2}{|c|}{ Aprimoramento/autoestima } & \\
\hline & \multicolumn{2}{|c|}{ Valores } & \\
\hline & \multicolumn{2}{|c|}{ Compreensão } & \\
\hline & \multicolumn{2}{|c|}{ Proteção } & \\
\hline & \multicolumn{2}{|c|}{ Carreira } & \\
\hline & \multicolumn{2}{|c|}{ Expectativa } & \\
\hline & \multicolumn{2}{|c|}{ Conflito } & \\
\hline \multirow{3}{*}{ 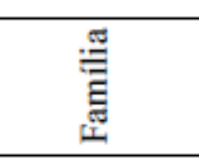 } & \multicolumn{2}{|c|}{ Responsabilidades } & \\
\hline & \multicolumn{2}{|c|}{ Apoio/suporte } & \\
\hline & \multicolumn{2}{|c|}{ Influência } & \\
\hline \multirow{6}{*}{ 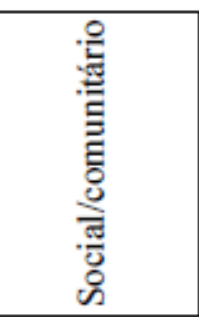 } & \multicolumn{2}{|c|}{ Socializar } & \\
\hline & \multicolumn{2}{|c|}{ Compromisso social } & \\
\hline & \multicolumn{2}{|c|}{ Reconhecimento } & \\
\hline & \multicolumn{2}{|c|}{ Apoio/suporte } & \\
\hline & \multicolumn{2}{|c|}{ Pertencimento } & \\
\hline & \multicolumn{2}{|c|}{ Agente de mudança social } & \\
\hline \multirow{16}{*}{ 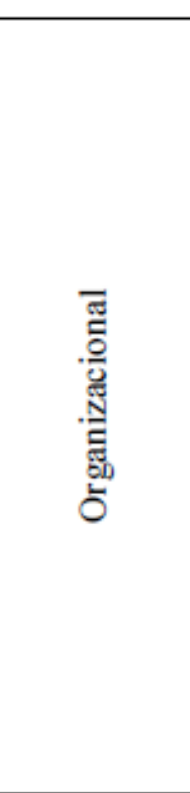 } & \multicolumn{2}{|c|}{ Infraestrutura } & \\
\hline & \multicolumn{2}{|c|}{ Serviços e atividades } & \\
\hline & Car & ga-horária & \\
\hline & Sele & ção & \\
\hline & Rec & rutamento & \\
\hline & Cap & acitação & \\
\hline & Rec & ursos humanos & \\
\hline & Ince & ntivos & \\
\hline & Aut & onomia & \\
\hline & Con & aunicação & \\
\hline & Rel & ações interpessoais & \\
\hline & $\mathrm{Apc}$ & io organizacional & \\
\hline & Est1 & essores & \\
\hline & Aco & mpanhamento & \\
\hline & Rel & ação com a comunidade & \\
\hline & $\begin{array}{l}\text { Rel } \\
\text { (gor }\end{array}$ & $\begin{array}{l}\text { ações externas } \\
\text { vernos/Instituições) }\end{array}$ & \\
\hline
\end{tabular}

Fonte: Elaboração própria 
O Quadro 1 representa a versão final do instrumento temático proposto por este estudo. Nele estão elencados os fatores temáticos e suas respectivas dimensões, que foram considerados importantes para a discussão sobre a retenção de voluntários sociais, na área da

saúde. Sua terceira coluna é composta pelo espaço destinado ao uso das instituições que queiram realizar pesquisas sobre a temática, para que cada organização elabore questões específicas, de acordo com o seu contexto, dentro de cada fator temático.

\subsubsection{Limitações do Estudo}

No tocante à análise e discussão dos resultados, ao se aplicar a análise de conteúdo com cunho qualitativo e tendo como suporte o processo de categorização semântica, incorre- se na possibilidade de erro na compreensão exata das informações extraídas dos artigos incluídos na revisão sistemática. $\mathrm{Na}$ realização do grupo focal, alguns participantes apresentaram dificuldades no preenchimento dos questionários e alegaram que, se o debate tivesse ocorrido primeiro, poderia ter facilitado o entendimento sobre alguns fatores. $\mathrm{O}$ grupo, ainda, gerou uma questão polêmica ao atribuir importância aos fatores políticos, mas não a sua respectiva dimensão (política). Tendo em vista que o tratamento e a conclusão dos dados só ocorreram após a elaboração do grupo, não foi possível realizar uma investigação mais profunda sobre esse resultado.

Há de se considerar, ainda, "a impossibilidade de fazer generalizações empíricas de seus resultados" (BUNCHAFT; GONDIM, 2004, p. 76), quando o processo de obtenção de dados parte da elaboração de um grupo restrito, no caso seis participantes, mesmo que composto por indivíduos especialistas da área em discussão.

\section{CONCLUSÃO}

Tiveram sua importância reconhecida as dimensões individual, familiar, social e organizacional. A dimensão política, que representa a relação externa entre as organizações de voluntariado com governos ou outras instituições, não obteve abordagem significativa nos artigos mapeados e nem foi considerada importante pelo grupo focal, não sendo, assim recepcionada na versão final do instrumento proposto.

Cada uma das dimensões apresenta fatores temáticos que podem exercer algum tipo de influência na decisão de permanência do voluntário. Com relação a construção do instrumento, concluiu-se pela seleção de 36 fatores temáticos, organizados nas quatro respectivas dimensões: 
individual (sociodemográficos, saúde do voluntário, experiência anterior com a doença, experiência com o voluntariado, aprimoramento/autoestima, valores, compreensão, proteção, carreira, expectativa, conflito); familiar (responsabilidades, apoio/suporte, influência); social (socializar, compromisso social, reconhecimento, apoio/suporte, pertencimento, agente de mudança social) e organizacional (infraestrutura, serviços e atividades, carga-horária, seleção, recrutamento, capacitação, recursos humanos,

incentivos, autonomia, comunicação interna, relações interpessoais, apoio organizacional, estressores, acompanhamento, relação com a comunidade, relações externas).

Da análise desses fatores se constatou a importância da existência de fatores individuais, que são abordados desde as teorias gerais de motivação, como a teoria das motivações humanas (MASLOW, 1943), até os modelos específicos do voluntariado, como o modelo motivacional multidimensional, criado por Clary et al. (1998). Também foram observados fatores relacionados à dinâmica familiar. Ademais, foram registrados fatores sociais, corroborando com estudos de base na teoria das trocas sociais. Assim como fatores relacionados à organização, reafirmando sua importância já considerada no modelo elaborado por Omoto e Snyder (1995).

Os fatores mapeados, religião, fé e econômico não foram inseridos na versão final do instrumento proposto, pois não tiveram relevância em nenhuma das duas fases da pesquisa. Um fato que chamou a atenção no decorrer deste estudo foi a ausência do fator liderança, uma vez que não foi observada a abordagem de temáticas ligadas à influência das lideranças no processo de motivação para a retenção dos voluntários.

Para estudos futuros, sugerem-se, então, novas investigações sobre a influência da imagem do líder no comportamento dos voluntários, assim como sobre as relações entre as organizações voluntárias e outras instituições e governos, resgatando a possibilidade da existência de uma dimensão política. Além disso, a elaboração de mais estudos sobre os estressores psicológicos ligados às atividades do voluntariado na saúde, tendo em vista que o grupo focal trouxe a informação da existência de vários tipos de situações complicadas. 


\section{REFERENCIAS}

AFARI-ASIEDU, S. et al. Volunteering for Health Services in the Middle Part of Ghana: In Whose interest? International Journal of Health Policy and Management, Kerman, v. 7, n. 9, p. 836-846, 2018.

ALAM, K.; TASNEEM, S.; OLIVERAS, E. Retention of female volunteer community health workers in Dhaka urban slums: a case-control study. Health Policy and Planning, [Oxford], v. 27, n. 6, p. 477-486, 2012.

ALAM, K.; TASNEEM, S.; OLIVERAS, E. Retention of female volunteer community health workers in Dhaka urban slums: a prospective cohort study. Human Resources for Health, [London], v. 12, p. 29, 2014.

BARDIN, L. Análise de conteúdo. Lisboa: Edições 70, 1977.

BECKHAUSER, S. P. R.; DOMINGUES, M. J. C. S. A profissionalização da gestão do voluntariado: um estudo de caso do departamento de voluntários do Hospital Israelita Albert Einstein. Saúde e Sociedade, São Paulo, v. 26, n. 4, p. 1026-1043, 2017.

BRAZ, R. P. Instrumento temático para avaliações sistêmicas dos fatores que influenciam a retenção de voluntários sociais na área da saúde. Niterói, 2019. 135 f. Dissertação (Mestrado em Sistemas de Gestão) - Universidade Federal Fluminense, Niterói, 2019.

BUNCHAFT, A. F.; GONDIM, S. M. G. Grupos focais na investigação qualitativa da identidade organizacional. Exemplo de aplicação. Revista Estudos de Psicologia, PUC, Campinas, v. 21, n. 2, p. 63-77, mai/ago, 2004.

CAMPBELL, J. et al. Managerial behavior, performance and effectiveness. New York, McGraw-Hill Book Company, 1970.

CHATIO, S.; AKWEONGO, P. Retention and sustainability of community-based health volunteers' activities: a qualitative study in rural Northern Ghana. Plos One, San Francisco, v. 12, n. 3, p. 1-12, 2017. DOI: https://doi.org/10.1371/journal.pone.0174002.

CLARY, E. G. et al. Understanding and assessing the motivations of volunteers: a functional approach. Journal of personality and social psychology, Washington, v. 74, n. 6, p. 15161530, 1998.

CLAXTON-OLDFIELD, S.; CLAXTON-OLDFIELD, J. Should I Stay or Should I Go: A Study of Hospice Palliative Care Volunteer Satisfaction and Retention. American Journal of Hospice and Palliative Medicine, Weston, v. 29, n. 7, p. 525-530, 2012.

CNAAN, R.; S. GOLDBERG-GLEN, R. Measuring Motivation to Volunteer in Human Services. Journal of Applied Behavioral Science, Thousand Oaks, v. 27, 3 ed., p. 269-284, 1991.

FERREIRA, M. J. R. L. Motivaçãoes e gestão do voluntariado no setor hospitalar em portugal. Porto, 2012. 238 f. Tese (Doutoramento em Ciências Empresariais) - Faculdade de Economia da Universidade do Porto, Porto, 2012. 
FRISCH, M. B.; GERRARD, M. Natural helping systems: A survey of Red Cross volunteers. American Journal of Community Psychology, Washington, v. 9, n. 5, p. 567-579, 1981.

GONDIM, S. M. G. Grupos focais como técnica de investigação qualitativa. Desafios metodológicos. Paidéia, Ribeirão Preto, v. 12, n. 24 p. 149-161, 2003. DOI: 10.1590/S0103863X2002000300004

HERZBERG, F. One more time: how do you motivate employees? Harvard Business Review, Boston, v. 81, n. 1, 87-96, 2003.

HOMANS, G. C. Social behavior: Its elementary forms. New York: Routledge \& Kegan Paul; 1961.

http://www.scielo.br/pdf/ress/v24n2/2237-9622-ress-24-02-00335.pdf. Acesso em 06 out. 2018. DOI: http://dx.doi.org/10.5123/S1679-49742015000200017.

HYDE, M. K. et al. Episodic Volunteering and Retention. Nonprofit \& Voluntary Sector Quarterly, Thousand Oaks, v. 45, n. 1, p. 45-63, 2016.

LIMA, G. B. A. Fatores temáticos e dimensões de influência do voluntariado social na área da saúde: uma revisão sistemática. In: CONGRESSO NACIONAL DE EXCELÊNCIA EM GESTÃO, 15, 2019, Rio de Janeiro. [No prelo].

LOBOS, J. Teorias Sobre a Motivação no Trabalho. RAE-Revista de Administração de Empresas, São Paulo, v. 15, n. 2, mar-abr, 1975.

LUDWICK, T. et al. Poor retention does not have to be the rule: retention of volunteer community health workers in Uganda. Health Policy and Planning, [Oxford], v. 29, n. 3, p. 388-395, 2014.

MASLOW, A. H. A theory of human motivation. Psychological Review, Washington, DC, v. 50, p. 370-396, 1943. Disponível em: https://psychclassics.yorku.ca/Maslow/motivation.htm. Acesso em: 08 ago. 2019.

MAYS, D. C. et al. Supporting and retaining Village Health Teams: an assessment of a community health worker program in two Ugandan districts. International Journal for Equity in Health, [London], v. 16, p. 1-10, 2017.

MEYER JUNIOR, V.; PASCUCCI, L.; MURPHY, P. Volunteers in Brazilian hospitals: good citizens or strategic agents? Voluntas, New York, v. 24, n. 2, p. 293-310, 2012.

MOGHADDAM, H. R.; ALLAHVERDIPOUR, H.; MATLABI, H. Successful recruitment and retention strategies for women health volunteers: viewpoints of the volunteers' supervisors and relevant researchers. Journal of Multidisciplinary Healthcare, [Auckland, N.Z.], v. 11, p. 621- 634, 2018.

MOHER D. et al. Principais itens para relatar Revisões sistemáticas e Meta-análises: A recomendação PRISMA. Tradução: Taís Freire Galvão e Thais de Souza Andrade Pansani; retro-traduzido por: David Harrad. Epidemiologia e Serviços de Saúde, Brasília, v. 24, n. 2 , p. 335-342, June 2015. Disponível em: 
OMOTO, A. M.; SNYDER, M. Sustained helping without obligation: Motivation, longevity of service, and perceived attitude change among AIDS volunteers. Journal of Personality and Social Psychology, Washington, v. 68, n. 4, p. 671-686, 1995. Doi: http://dx.doi.org/10.1037/0022-3514.68.4.671

PÉREZ-RAMOS, J. Motivação no trabalho: abordagens teóricas. Psicologia USP, São Paulo, v. 1, n. 2, p. 127-140, 1990.

RODRIGUES, K. M.; MEYER JUNIOR, V.; CRUZ, J. A. W. Trabalho voluntário e seu gerenciamento: desafios para um hospital comunitário. Revista de Administração

Hospitalar e Inovação em Saúde, Belo Horizonte, v. 11, n. 4, p. 306-323, 2014.

SANOU, A. K. et al. Motivation of Community Health Workers in Diagnosing, Treating, and Referring Sick Young Children in a Multicountry Study. Clinical infectious diseases: an official publication of the Infectious Diseases Society of America, Chicago, v. 63, n. 5, p. 270-275, 2016.

SARTI, A. J. et al. Exploring the components of physician volunteer engagement: a qualitative investigation of a national Canadian simulation-based training programme. BMJ Open, [London], v. 7, n. 6, 2017. Disponível em:

http://bmjopen.bmj.com/content/7/6/e014303.abstract.

SILVA, C. P. R.; CONCEIÇÃO, A. P.; CHAGAS, A. P. S. Clown: o palhaço como intervenção e humanização em saúde. Journal Health Biology Science, Fortaleza, v. 5, n. 4, p. 352-359, 2017.

SINGH, D. et al. Motivation of Community Health Volunteers in rural Uganda: the interconnectedness of knowledge, relationship and action. Public Health, London, v. 136, p. 166-171, 2016.

SNYDER, M.; OMOTO, A. M. Volunteerism: Social Issues Perspectives and Social Policy Implications. Social Issues and Policy Review, Medford, v. 2, n. 1, p. 1-36, 2008.

STRACHAN, D. L. et al. Interventions to improve motivation and retention of community health workers delivering integrated community case management (iCCM): stakeholder perceptions and priorities. The American journal of tropical medicine and hygiene, Baltimore, v. 87, p. 111-119, 2012. Suppl 5

TAMAYO, A.; PASCHOAL, T. A relação da motivação para o trabalho com as metas do trabalhador. Revista de Administração Contemporânea, Maringá (PR), v. 7, n. 4, p. 33-54, 2003.

TOPP, S. M. et al. Motivations for entering and remaining in volunteer service: findings from a mixed-method survey among HIV caregivers in Zambia. Human Resources for Health, [London], v. 13, n. 1, p. 72, 2015.

VERGARA, S. C. Métodos de pesquisa em administração. 6. ed. São Paulo: Atlas S. A., 2015.

VERGARA, S. C. Projetos e relatórios de pesquisa em administração. 16. ed. São Paulo: Atlas, 2016. 
O INOVAÇÃo

WIDJAJA, E. Motivation Behind Volunteerism. 2010. Completion of course work (Bachelor of Arts) - Claremont McKenna College, Claremont, 2010. Disponível em: https://scholarship.claremont.edu/cmc_theses/4/. Acesso em: 12 out. 2018.

WILSON, J. Volunteering. Annual Review of Sociology, Palo Alto, v. 26. p. 215-240, 2000. DOI: https://doi.org/10.1146/annurev.soc.26.1.215 